\title{
Carnets
}

Revue électronique d'études françaises de l'APEF

Première Série - 5 | 2013

Métamorphoses littéraires

\section{La métamorphose de l'image chez Yves Bonnefoy: Le mouvement du poème}

\section{Márcia Marques Rambourg}

\section{OpenEdition}

1 Journals

\section{Edição electrónica}

URL: http://journals.openedition.org/carnets/8351

DOI: $10.4000 /$ carnets.8351

ISSN: 1646-7698

\section{Editora}

APEF

Edição impressa

Data de publição: 1 Maio 2013

Paginação: 131-141

\section{Refêrencia eletrónica}

Márcia Marques Rambourg, « La métamorphose de l'image chez Yves Bonnefoy: Le mouvement du poème », Carnets [Online], Première Série - 5 | 2013, posto online no dia 23 junho 2018, consultado o 01 maio 2019. URL : http://journals.openedition.org/carnets/8351 ; DOI : 10.4000/carnets.8351

\section{(). $(1) \Theta$}

Carnets est mis à disposition selon les termes de la licence Creative Commons - Atribution - Pas d'utilisation commerciale 4.0 International. 


\title{
LA MÉTAMORPHOSE DE L'IMAGE CHEZ YVES BONNEFOY \\ Le mouvement du poème
}

\author{
MÁRCIA MARQUES RAMBOURG \\ Université Paris IV, CRIMIC \\ mmrambourg@gmail.com
}

\begin{abstract}
Resumo: Neste artigo, tentamos estudar, brevemente, a noção de imagem poética na obra do poeta francês contemporâneo, Yves Bonnefoy. Tal noção é abordada sob a égide do movimento e da ação do ato da criação e da recriação poéticas. Se a poesia de Yves Bonnefoy exalta a percepção da imagem poética como produtora de outras imagens, de outros "países", ela buscará investigar, de mesma maneira, os mecanismos de movimento e de transformação desta mesma imagem.
\end{abstract}

\begin{abstract}
In this article, we attempt to examine, briefly, the notion of poetic image in the work of contemporary French poet Yves Bonnefoy. This notion will be discussed under the perspective of the movement and action of creation and recreation in Poetics. If the work of Yves Bonnefoy exalts the perception of the poetic image as a producer of other images, other "countries", it will seek to investigate, in the same way, the mechanisms of movement and transformation of that image.
\end{abstract}

Palavras-chave: Imagem, fenomenologia, paisagem, metamorfose

Keywords: Image, phenomenology, landscape, metamorphosis 
Dessiner, dé-signer. Briser le sceau, ouvrir l'enveloppe, - mais elle reste fermée. Peindre, alors: laisser le monde, toutes ses rives tous ses soleils, tous ses vaisseaux glissant 'dans l'or et dans la moire’ se refléter dans la vitre.

Yves Bonnefoy

\section{Introduction}

Yves Bonnefoy est une référence dans la poésie française contemporaine de par sa contribution au paysage esthétique et critique de celle-ci. L'ensemble de son œuvre poétique constitue un important panorama de la littérature francophone et mondiale. S'organisant sous l'influence surréaliste, la poétique de Bonnefoy évolue esthétiquement dans de nouvelles approches de style et de problématiques questionnant la mort, l'Autre, Dieu, la limite entre Ici et Ailleurs, et l'image poétique, en tant que recherche spatiale.

Cet article propose une lecture de l'image poétique de l'œuvre bonnefoyienne en tant qu'espace mouvant, ouvert et évocateur d'autres horizons. Cette perspective du changement (et du mouvement) du signe poétique constitue le point de départ de notre argumentation sur la métamorphose de l'image dans l'œuvre du poète français.

Tout d'abord, observons que l'image poétique renvoie à un discours, de façon plus ample. Celui-ci (en tant que discours littéraire) peut contenir, comme nous pouvons observer dans Approches de la réception, de Georges Molinié et de Alain Viala, "trois composantes définitionnelles" (Molinié, Viala, 1993:17).

Pour la première de ces composantes, le discours constitue son propre système sémiotique, en quatre partitions: "la substance du contenu, la forme du contenu, la forme de l'expression, la substance de l'expression" (ibid.). II est, en outre, "bien en lui-même une totalité de fonctionnement sémiotique, qui régule entièrement, et dualement, sur son propre système" (id.: 19).

Ensuite, le discours littéraire est son propre référent et développe au sein de sa propre structure un système sémiotique pragmatique et performatif:

prenons le cas d'un roman de Zola. On peut résumer d'une part l'enregistrement des conditions sociales (matérielles et mentales) de vie des ouvriers dans tel endroit à telle époque, d'autre part l'expression des sentiments divers de représentation contemporains d'autres catégories sociales face à un milieu dépeint; on peut enfin condenser une argumentation tendant à faire prendre conscience au plus grand nombre de la situation, pour favoriser une évolution améliorative: point de littérature.[...] 
Mais le roman de Zola comme roman, le discours romanesque de Zola comme littéraire, définit une création qui, en tant que romanesque, en tant que littéraire, n'a pas pour référent ces ingrédiens qu'on vient d'énumérer, mais un objet particulier de nature tout everbale, qui est à soi seul un être du monde: un roman (id.: 21).

Finalement, pour la troisième composante définitionnelle le discours littéraire, il "se réalise dans l'acte de désignation de l'idée de ce référent". II se "définit ainsi, toujours dans une perspective pragmatique, à un degré avancé, ou décalé" (id.: 22). Le discours littéraire fait "apparaître l'idée du référent dans son propre déroulement". Il est réflexif, il contient l'idée de l'autoréférence.

Dans cette perspective de la Sémiostylistique que nous venons de citer, la troisième composante du discours littéraire est la plus pertinente dans notre proposition d'étude sur l'image poétique chez Yves Bonnefoy.

En ce qui concerne la structure interne du poème de Bonnefoy, il est intéressant d'envisager l'idée de désignation autotélique: comment les éléments de syntaxe constituent le travail de référence sémiotique. II est également relevant d'imaginer le modus operandi génétique de la poétique bonnefoyienne sous cet angle structuraliste où l'analyse structurelle de la fonction de l'image poétique n'est jamais excessive.

Cet article propose, pourtant, une lecture basée plutôt sur la description sémiotique du discours littéraire et le mouvement de ce discours référentiel, performatif.

Comme nous rappelle Michel Collot, Yves Bonnefoy "comme plusieurs des poètes et des peintres rassemblés un moment autour de la revue I'Ephémère, a toujours défendu et illustré une poétique et une esthétique transitives, animées du désir d'ouvrir l'œuvre, autant que possible, au monde extérieur" (Collot, 2005).

Nous parvenons ainsi à la notion d'horizon en Poésie. À cette notion, se relie celle de phénomènes, des horizons "éveillés avec tout donné réel” (Husserl, 1970: 97).

La poésie de Bonnefoy évoque la problématique de l'horizon, du phénomène de l'image de la Parole poétique qui s'éveille dans un "horizon d'indétermination déterminable" ou d'un "horizon de déterminabilité indeterminé", comme nous invite Michel Collot à relire la phénoménologie de Husserl (Collot, 2005: 21).

Cette opération phénoménologique que nous retrouvons dans le texte de Bonnefoy est une des perspectives de son travail poétique où la Parole est un paysage en formation, un monde-image avant la langue, avant l'actualisation d'un espace possible:

Le vrai commencement de la poésie, c'est quand ce n'est plus une langue qui décide de l'écriture, une langue arrêtée, dogmatisée, et qui laisse agir ses structures propres; mais quand s'affirme au travers de celles-ci, relativisées, littéralement démystifiées, 
une force en nous plus ancienne que toute langue; une force, notre origine, que j'aime appeler parole (Bonnefoy, 1990: 33).

Lors d'un entretien avec Bernard Falciola, Yves Bonnefoy nous illustre son idée de monde et d'organisation des paysages poétiques:

Le monde que nous recevons [...] de ce qui en nous questionne l'être au-dehors, qu'est-ce qu'est au juste? La rencontre de présences élémentaires que nous tenons pour réelles - les fruits, les arbres, quelques êtres, quelques façons d'exister - et des mirages comme en forment dans tout psychisme les aspirations instinctives, les préjugés, les refus: un total, une rêverie, où ces fruits, ces arbres, mais les montagnes aussi, et telle sorte de pierre, et la huppe qui vole sur les rochers comme une fée travestie, et nos proches et toutes nos valeurs, toutes nos croyances, se sont recomposés en une figure, qui, s'il n'y avait pas l'élaboration vraiment poétique [...], refléterait peut-être surtout mon refus à la finitude (id.: 28).

L'image sera ainsi le silence performatif du paysage, ce qui l'actualisera dans les possibilités et dans les changements de celui-ci:

Par 'image', j'entendais et j'entends toujours, non certes le simple contenu de la perception, ni même les représentations qui se forment dans notre rêverie, lesquelles sont fugitives: mais ce que Baudelaire avait en esprit quand il évoquait 'le culte des images, ma grande, mon unique, ma primitive passion', et ce que Rimbaud désignait, lui aussi, quand il écrivait dans un poèmes des Illuminations, le poème 'Après le Déluge': 'Dans la grande maison de vitres encore ruisselante, les enfants en deuil regardèrent les merveilleuses images' [...]. Les images, c'est le cadre, la page, la fixité du tracé, tout ce qui semble faire de la vision fugitive un fait malgré tout, un fait relevant d'un autre lieu que celui de notre vie, et témoignant même peut-être de l'existence d'un autre monde (id.: 12).

\section{Le changement de l'image poétique}

La notion de changement - ou la "perception du changement" pour emprunter le terme à Henri Bergson - est une notion-clef dans la définition de métamorphose de l'image poétique que nous venons d'entrevoir de l'œuvre d'Yves Bonnefoy.

Le changement, en tant concept, établit un problème. En tant qu'observation pragmatique, il se définit davantage comme fait observé. Lorsqu'on observe le changement d'un quelconque objet dans l'espace, l'on évoque son état définitif, ou le résultat de son 
expérience dans le temps. Nous ne "pensons" pas au changement: nous le "constatons", dans le résultat des choses.

Pourtant, le changement qui est "constitutif de toute notre expérience" (Bouaniche, 2011: 18) constitue plus un problème qu'un résultat. Car cet ensemble d'états accidentels de l'expérience relève d'un processus évolutif qui, dans l'opération intellectuelle que l'on relie à un objet dans l'espace, il sera en continuelle relation avec d'autres notions.

II s'agit d'un problème qui implique la notion d'intuition et celle d'espace-temps. Relevant, enfin, de toute expérience, le changement s'établit dans une durée déterminée dans la relation avec d'autres changements. Et parce qu'il garde son aspect d'indivisibilité et de substantialité, il est lié à la mémoire. Le changement est donc cette opération à deux versants - conceptuel et empirique - qui implique notre perception de l'espace et du temps.

Le poème, qui est un espace de travail sémiotique, sera un lieu de changement et de transformation. Si nous envisageons cette dynamique du changement en tant que perception, application et entendement de l'espace mouvant, et surtout en tant qu'acte de mémoire, et de présence, le poème sera alors un espace en constante ouverture, changeant, dialogique et conservateur d'un passé et d'une substance. Traversant notre vision des choses, il forme alors une mémoire de lecture, un passé dans le présent.

Cette mémoire de lecture, qui est ouverte et mouvante, est une sphère importante dans la poétique de Bonnefoy. Le "sommeil" de la poésie, de la parole minérale, encore non dite, l'état de veille du verbe, le lieu, donc, de changement et de transformation témoigne d'un travail initial de construction de l'image; d'une réflexion importante sur la capacité qu'a celle-ci de changer et de se transformer de se reconstruire.

Pour Yves Bonnefoy, les images signifient "moins le désir de représenter notre monde que celui d'en bâtir un autre". Ce besoin sémiotique à deux versants est à l'origine, en effet, d'un étant poétique:

Et le poème, s'il a 'tenu' une fois, dans l'exigence sévère d'une poésie qui se forme, vaudra donc, et durablement, pour celui qui l'apprécie et ne cesse d'y revenir; sauf que ce dernier ne lira plus jamais de la même façon d'une année à l'autre: il change, lui aussi, et fait devenir ce qu'il lit, ce qu'il peut même savoir par cœur [...] Cette remise en question, cette table rase, serait-elle pour un instant seulement, c'est elle le 'silence' [...] C'est le moment le plus véridique du travail de la poésie; et il n'y a de vraie création à mes yeux que sui le silence de l'origine peut se maintenir, d'une certaine façon, dans la nouvelle écriture (id.: 24).

Le silence de l'écriture est la dimension spirituelle des choses dans notre perception; la tension métaphysique et empirique entre le passé et le présent. II constitue un espace 
vide, un lieu de création dans lequel les structures de signification s'établissent et s'organisent dans de nouveaux horizons créateurs.

À la lecture de "Une pierre", du chapitre éponyme du recueil La vie errante, nous lisons:

\author{
J'ai toujours faim de ce lieu \\ Qui nous était un miroir, \\ Des fruits voûtés dans son eau, \\ De sa lumière qui sauve, \\ Et je graverai dans la pierre \\ En souvenir qu'il brilla \\ Un cercle, ce feu désert. \\ Au-dessus le ciel est rapide
}

Comme au vœu la pierre est fermée.

Que cherchions-nous? Rien peut-être,

Une passion n'est qu'un rêve,

Ses mains ne demandent pas,

Et de qui aima une image,

Le regard a beau désirer,

La voix demeure brisée,

La parole est pleine de cendres (Bonnefoy, 1993:103).

Le choix lexical de la première strophe évoque une structure analogique où "lieu", "miroir", "eau" et la proposition subordonnée "qui sauve" se relient en introduisant une lecture anaphorique. Celle-ci déployée le long des quatre strophes, va alors établir les thèmes suivants, épistrophiques à leur tour, à reprendre: la "pierre", le "miroir", le "cercle, ce feu désert", l' "image", "la voix brisée", enfin, "la parole". Cette séquence d'images constitue ici une perspective surréaliste qui se repose sur deux axes essentiels - celui de souvenir, de ce qui renvoie à l'origine de l'expérience du texte ("Et je graverai dans la pierre/ En souvenir qu'il brilla/ Un cercle, ce feu désert") - et celui de transformation et d'ouverture, de "faim" de nouveaux paysages, un retour au silence, un lieu à réinventer, à refaire ("Et de qui aima une image,/Le regard a beau désirer,/La voix demeure brisée,/La parole est pleine de cendres").

Le changement est, ainsi, dans la poétique bonnefoyienne, un lieu ouvert; un horizon investigateur qui dessine et dé-signe l'image, son essence et son application poétique.

"Le désespoir du peintre", du chapitre "Encore les raisins de Zeuxis", du recueil La vie errante, est représentatif de cette double fonction de l'image poétique, où nous observons le 
sentiment d'absence du monde et de sa transformation, et la tentative d'ancrer le réel dans l'expérience subjective. La peinture, objet du monde, est ici dramatique; elle est action, scène, description et évolution. Indéfinie, elle devient objet-monde dans l'actualisation de l'art, et se dissipe, évoquant le deuil d'un tableau-monde désormais réduit à un "tas de blocs de houille luisante":

II peignait, la pente d'une montagne, pierres ocres serrées, mais cette étoffe de bure se divisait, pour un sein, un enfant y pressait ses lèvres, et on descendait, de là-haut, de presque le ciel, dans la nuit (car il faisait nuit), c'étaient des porteurs de coffres desquels filtraient des lumières.

Que des tableaux laissa-t-il ainsi, inachevés, envahis! Les années passèrent, sa main trembla, l'oeuvre du peintre de paysage ne fut que ce tas de blocs de houille luisante, là-bas, sur quoi erraient les enfants du ciel et de la terre (id.:70).

Il est, ainsi, important d'observer que cet espace mouvant entre mémoire et présent, entre être et devenir est un espace d'expérience, ce sont des "tableaux inachevés, envahis". Le changement qui s'opère dans la poésie d'Yves Bonnefoy témoigne de la façon dont le mouvement se fait dans le mouvement, le temps dans le temps; et l'image dans la possibilité des images. Dans cette approche métamorphique, la notion même de transformation et de silence - rappelons ici, l'instant entre le je-ne-sais-quoi et le presque-rien ${ }^{1}$ - nous renvoie à une tension perpétuelle, en constant appel à l'expérience du texte. Nous retrouvons, tout au long de l'œuvre poétique d'Yves Bonnefoy, des intervalles fertiles d'un instant-parole, où s'opère la transformation de l'image, l'éveil du verbe en état minéral. S'impose, ainsi, et de façon non exhaustive, la lecture de Du mouvement et de l'immobilité de Douve et de Pierre écrite. Ces deux recueils nous interpellant dans ce que l'image poétique peut évoquer; dans la capacité que celle-ci a de se transformer elle-même, dans des mots et des mondes, comme nous verrons plus loin.

\section{La métamorphose de la pierre}

Je ne doute pas que je puisse dessiner, comme en creux dans le langage conceptuel, le schéma de ce qui n'est pas. Mais ce néant du concept doit être plus qu'une virtualité. [...] Nul problème

\footnotetext{
1 Nous nous référons ici à la philosophie métaphysique de Vladimir Jankélévitch laquelle, dans la même perspective que celle d'Henri Bergson, établit une pensée spatio-temporelle "ouverte" et dialectique.
} 
ne peut favoriser la métamorphose, rien non plus ne saurait l'empêcher.

Yves Bonnefoy

Depuis les études de la Phénoménologie chez d'importants philosophes comme Husserl, Levinas, Sartre et Merleau-Ponty, il nous est possible d'approfondir notre lecture du monde: la façon dont nous le percevons, le recevons et l'organisons. Cette organisation mentale qui se donne corporelle et spirituellement fera de nous des sujets d'un monde que nous devons arranger; des organisateurs de l'espace à la fois actifs et passifs; percevants et perçus. Le monde que nous nous efforçons de spatialiser sera, à son tour, spatialisant et organisateur.

Ce monde qui est ainsi fait de répétitions, d'identifications et de relations est une masse hétérogène de lectures. II se forme autour des valeurs sociales spécifiques. Une fois formé, il se communique avec d'autres mondes, avec d'autres valeurs et avec d'autres bases sémiotiques, à partir d'une logique rhétorique qu'est la suppression et la supplémentation des éléments de cette réalité:

Pour faire un monde à partir d'un autre, il faut souvent procéder à des coupes sévères et à des opérations de comblement - à l'extraction véritable de vieux matériaux et à leur remplacement par de nouveaux. Notre capacité à laisser échapper est virtuellement illimitée, et ce que nous appréhendons, ce sont habituellement des fragments significatifs et des repères qui nécessitent des compléments massifs [...] Dans la pénible situation d'avoir à relire des épreuves [...], nous passons immanquablement sur quelque chose qui est là et voyons quelque chose qui n'est pas là (Goodman, 1992: 33).

Or, le monde poïétique est un espace organisé en fonction des possibilités sémiotiques et surtout "trans-sémiotiques" (Molinié, 1998: 43 -121). II est ainsi un réel en mouvement qui nous parle et qui nous spatialise dans son silence organisationnel. Dans cette dynamique phénoménologique du monde poétique, ce qui nous importe d'observer est le processus de médiation des mondes, d'actualisation des possibles, c'est-à-dire des espaces en puissance, avant même de réaliser le résultat de cette métamorphose.

La métamorphose chez Yves Bonnefoy, de par le travail de médiation et de création du texte poétique, sera, enfin, ce lieu transitionnel, d'espace entre image et après-image.

Les recueils Du mouvement et de l'immobilité de Douve et Pierre écrite peuvent se réunir dans ce mouvement d'écriture. Le premier s'organise sur cinq sections, ou thématiques: "Théâtre", "Derniers Gestes", "Douve Parle", "L'orangerie", et "Vrai Lieu". Ce recueil évoque la quête de liberté et de mouvement du verbe poétique. 
Dans le poème "Vrai nom", de la deuxième section du même recueil, "Derniers Gestes", nous observons la recherche déictique du signe poétique: le besoin de montrer ce qui n'est pas; ce qui est ailleurs. Le besoin de dévoiler; de nommer l'innommable:

Je nommerai désert ce château que tu fus,

Nuit cette voix, absence ton visage,

Et quand tu tomberas dans la terre stérile

Je nommerai néant l'éclair qui t'a porté

\section{$[\ldots]$}

Je te nommerai guerre et je prendrai

Sur toi tes libertés de la guerre et j'aurai

Dans mes mains ton visage obscur et traversé,

Dans mon cœur ce pays qu'illumine l'orage (Bonnefoy, 1978: 51).

Comme d'autres poèmes qui illustrent cette idée de la fonction déictique de la poésie, dont "Cette pierre ouverte est-toi, ce logis dévasté", ou "Que saisir sinon qui s'échappe", "Vrai Nom" dialogue avec d'autres voix de l'œuvre de Bonnefoy dans cette nécessité de montrer ce que l'image immédiate, du monde, doit montrer en poésie: l'au-delà du monde; le dehors. Au-delà du "je", la définition et le nom sous-jacent: "Je nommerai désert ce château que tu fus/ [...] Je nommerai néant l'éclair qui t'a porté/ [...] Je te nommerai guerre et je prendrai ".

C'est le cas de "Vrai Lieu", dernière section de Du mouvement et de l'immobilité de Douve, qui nous amène à un silence mouvant des images, vers un espace présent dans la distance de ces images: "Qu'une place soit faite à celui qui approche, /Personnage ayant froid et privé de maison./Personnage tenté par le bruit d'une lampe, /Par le seuil éclairé d'une seule maison" (id.: 85).

Dans un rapport dialogique avec Du mouvement et de l'immobilité de Douve, Pierre écrite (recueil composé de quatre sections, dont "L'Eté de Nuit", "Pierre Ecrite", "Un Feu Va Devant Nous" et "Le Dialogue d'Angoisse et de Désir") évoque les déplacements et les répétitions, les mouvements et l'immobilité de l'image poétique. Déplacement spatial car l'image est en continuelle problématique entre Ici et Ailleurs, entre "Une Pierre" et "Le Lieu des Morts", entre ce qui "accable mon corps" et "le pli de l'étoffe rouge". Répétitions et immobilité car le mouvement que nous nous devons d'observer dans ce chapitre du recueil repose sur l'observation des différences et des (re)marques de déplacements: "Tombe, mais douce pluie, sur le visage./ Eteins, mais lentement, le très pauvre chameil." 
Il nous semble important d'observer que l'allégorie de la pierre, ce lieu originel de transformation à s'éveiller paradoxalement dans le sommeil, dans l'immobilité, se multiplie et se reconstruit, fertile, en d'autres terres, en d'autres lieux pierreux "de sommeil jeté sur la pierre" (Naughton, 1998: 47). L'immobilité qu'évoque l'image de la pierre est, ainsi, à la fois façonnée par l'immobilité et par le mouvement, par un souci de composition où les choses sont à découvrir, à montrer et à démontrer. C'est une terre "qu'il faut reconquérir presque à tout moment, tant peuvent ressurgir le doute, l'angoisse, le sentiment de la perte" (Naughton, 1998: 48). Cette terre errante qui est ainsi la "pierre poétique" chez Bonnefoy pressent une "philosophie de la composition" au rebours de celle chez Edgar Allan Poe, comme nous rappelle Michel Collot: "Il ne s'agit jamais, en poésie, de réaliser par l'écrit un projet de signification préalablement formé, ou d'exprimer une émotion ou une expérience déjà faite, mais de partir à la découverte" (Collot, 1992: 124).

Dans la poésie de Bonnefoy, la Parole engage le mouvement final d'un projet. Le processus, l'en-train-de du discours poétique, dans son mouvement, est à observer davantage dans son travail poétique.

Cette brève étude parcourt le "silence d'un ravin", l'image poétique des paysages possibles, l'inscription d'une pierre mouvante, où "la terre se dérobe", où le silence refait le monde, et les chemins au-delà de l'image. Dans la perspective du travail poétique chez Yves Bonnefoy, rien ne s'opère sans le changement et le mouvement des paysages:

\author{
Souvent dans le silence d'un ravin \\ J'entends (ou je désire entendre, je ne sais) \\ Un corps tomber parmi des branches. Longue et lente \\ Est cette chute aveugle; que nul cri \\ Ne vient jamais interrompre ou finir.
}

Je pense alors aux processions de la lumière

Dans le pays sans naître ni mourir (Bonnefoy, 1978: 106).

Le son du mot imagé, imaginé, tombe dans notre propre façon de voir [dans] le poème. Suggérant un espace ouvert, le silence du poème nous invite à la construction de celui-ci, à la métamorphose, à l'abri de l'écriture: "J'entends (ou je désire entendre, je ne sais)".

La "chute aveugle" de la lecture du texte bonnefoyien s'établit alors dans la métamorphose perpétuelle du signe du poème. Le texte de Bonnefoy se forme dans cet espace transitoire et transitionnel qu'est la la Parole, dans ce pays[age] qui s'annonce dans des éléments indéfinis, occultant et dévoilant le mouvement du travail poétique. 


\section{Bibliographie}

Bonnefoy, Yves (1993). La Vie errante. Paris: Poésie Gallimard. (1990). Entretiens sur la poésie (1972-1990). Paris: Mercure de France. (1992). "Enchevêtrements d'Ecriture: Entretien avec Michel Collot". In:Genesis 2: 124.

(2005). Yves Bonnefoy, Lumière et nuit des images, suivi de "Ut pictura poesis" et "D'autres remarques". Sous la direction de Murielle Gagnebin. Paris: Champ Vallon. 1998). Yves Bonnefoy. Cahier Onze. Sous la direction de Jacques Ravaud. Paris: Le Temps qui Fait. 2007). L’Arrière-Pays. Paris: Poésie Gallimard. 1978). Poèmes. Paris: Mercure de France. (2001). Les Planches courbes. Paris: Mercure de France.

Bergson, Henri (1896, 2008). Matière et mémoire. Paris: PUF, "Quadrige". BOUANICHE, Arnaud (2011). La perception du changement. Édition critique, sous la direction de Fédéric Worms. Paris: PUF.

Collot, Michel (2005). "Pays, imager, paysage". In: Yves Bonnefoy, Lumière et nuit des images, sous la direction de Murielle Gagnebin, Edition Champ Vallon: 115. (2005). La Poésie moderne et la structure d'horizon. Paris: PUF. (2005). Paysage et poésie, du romantisme à nos jours. Paris: José Corti.

Goodman, Nelson (1992). Manières de faire des mondes. Paris: Folio Essais. JANKELEVITCH, Vladimir (1957). Le Je-ne-sais-quoi et le presque-rien, Paris: PUF. HUSSERL, Edmund (1970). Expérience et Jugement. Paris: PUF. MeRleau-PonTy, Maurice (1945). Phénoménologie de la perception. Paris: Gallimard. (1964). Le visible et l'invisible. Paris: Tel Galliamard.

MOLINIÉ, Georges (1998). Sémiostylistique. L'effet de l'art. Paris: PUF. et VIALA, Alain (1993). Approches de la réception. Paris: PUF.

NAUGHTON, John. (1998). "Yves Bonnefoy: l'idée nécessaire de l'être". In: Yves Bonnefoy. Cahier onze sous la direction de Jacques Ravaud. Paris: Le Temps qu'il Fait, pp. 44-51.

STAROBINSKI, Jean (1982). "Yves Bonnefoy: la poésie entre deux mondes". In: Critique, № 350,1979 , repris en préface à Poèmes, Paris: Poésie/Gallimard. 